\title{
Selecting highly-compact radio sources for the definition of the celestial reference frame
}

\author{
Patrick Charlot $^{1}$, Alan L. Fey ${ }^{2}$, Roopesh Ojha ${ }^{2}$, David A. Boboltz ${ }^{2}$, \\ J. I. B. Camargo ${ }^{3}$ and A. Collioud ${ }^{1}$ \\ ${ }^{1}$ Observatoire de Bordeaux (OASU) - CNRS/UMR 5804, BP 89, F-33270 Floriac, France \\ email: charlot@obs.u-bordeaux1.fr \\ ${ }^{2}$ Earth Orientation Department, U.S. Naval Observatory (USNO), \\ 3450 Massachusetts Avenue NW, Washington, DC 20392-5420, USA \\ email: afey,rojha,dboboltz@usno.navy.mil \\ ${ }^{3}$ Observatório do Valongo, UFRJ, Lad. Pedro Antonio 43, Rio de Janeiro, RJ 20080-090, Brasil
}

\begin{abstract}
We discuss the issue of selecting highly-compact radio sources for the definition of the celestial reference frame.
\end{abstract}

Keywords. astrometry, reference systems, radio continuum: general, techniques: high angular resolution, techniques: interferometric, galaxies: active

The intrinsic radio structure of the extragalactic sources is one of the limiting errors in the definition of the International Celestial Reference Frame (ICRF). Based on multi-epoch VLBI images obtained with the Very Long Baseline Array and other VLBI telescopes around the world between 1994 and 2005, we evaluate this effect for 560 ICRF sources (about $80 \%$ of the current frame) and calculate a so-called 'structure index' to define the astrometric suitability of the sources.

The structure index ranges from 1 for the most compact sources to 4 for the most extended sources. The number of epochs for which the structure index is available for a given source varies from 1 for the least-observed sources to 20 for the intensively-observed sources. From this calculation, we identify a subset of 221 ICRF sources which have very good or good astrometric suitability (i.e., a structure index of either 1 or 2) at any of the available epochs.

We argue that these compact sources are potential candidates for defining the celestial frame with the highest accuracy when a future realization of the ICRF is made. 